\title{
Speeded up surveillance video indexing and retrieval using abstraction
}

\begin{abstract}
Many researches have been conducted on video abstraction for quick viewing of video archives, however there is a lack of approach that considers abstraction as a pre-processing stage in video analysis. This paper aims to investigate the efficiency of integrating video abstraction in surveillance video indexing and retrieval framework. The basic idea is to reduce the computational complexity and cost of overall processes by using the abstract version of the original video that excludes unnecessary and redundant information. The experimental results show a significant reduction of $87 \%$ in computational cost by using the abstract video rather than the original video in both indexing and retrieval processes.
\end{abstract}

Keyword: Video abstraction; Surveillance video; Video indexing; Video retrieval; Video indexing and retrieval 